\title{
PADRÃO ESPACIAL DA COMUNIDADE DE MACROALGAS DE MESOLITORAL EM AMBIENTE RECIFAL DO NORDESTE BRASILEIRO
}

Recebido em: 04/03/2013

Aceito em: 01/09/2013

\author{
Edson Regis Tavares Pessoa Pinho de VASCONCELOS ${ }^{1^{*}}$ \\ Thiago Nogueira de Vasconcelos REIS ${ }^{1}$ \\ Nathalia Cristina GUIMARÃES-BARROS ${ }^{1}$ \\ Juliane BERNARDI ${ }^{1}$ \\ Arsenio José ARECES-MALLEA ${ }^{1}$ \\ Adilma de Lourdes Montenegro COCENTINO ${ }^{1}$ \\ Mutue Toyota FUJII ${ }^{2}$
}

\section{ABSTRACT}

Due to many biotic and abiotic factors that affect the organisms distribution in the intertidal zone, this study assessed the intertidal benthic seaweed distribution and zonation in a reef section of Brazilian northeast. The data were obtained from transects taken in right angle to the coastline, during three sampling efforts in dry and rainy seasons. The frequency of occurrence of some algae such as Centroceras sp., Gelidiella acerosa, Laurencia dendroidea, Palisada perforate, Padina sp Sargassum sp., Sphacelaria sp. and Ulva sp. represent their capacity to tolerate environmental stress, which they are susceptible. The most frequent macroalgae can be found at any place along the $10 \mathrm{~m}$ transect. However there is a preference for some zones where the algae are grouped forming two distinct zones during the dry season. In the rainy season there is a loss of this

patterne.

Key words: algae zonation, diversity, benthic distribution

\section{RESUMO}

Frente a diversos fatores bióticos e abióticos que afetam a distribuição dos organismos na zona entremarés, o presente estudo avaliou a distribuição e a zonação das macroalgas bentônicas do mesolitoral em um trecho recifal do nordeste brasileiro. Os dados foram obtidos a partir de transecções traçadas perpendiculares à linha de costa, durante três campanhas amostrais no período seco e chuvoso. A freqüência relativa de algumas algas tais como Centroceras sp.,
Gelidiella acerosa, Padina sp., Palisada perforata e Ulva sp. representa bem a capacidade de tolerância ao estresse ambiental ao qual estão sujeitas, visto que elas apresentam ampla distribuição espacial e temporal ao longo do transecto de $10 \mathrm{~m}$. Entretanto, durante período seco estes táxons são responsáveis pela formação de dois grupos em função do gradiente de distância analisado e no período chuvoso há perda deste padrão.

Palavras chave: algas, zonação, diversidade, distribuição do bentos

\section{INTRODUÇÃO}

Diversos fatores abióticos (e.g. variação do nível de maré, grau de exposição às ondas, inclinação do substrato, dessecação e sombreamento) e bióticos (e.g. predação, herbivoria e competição) são conhecidos por afetar a distribuição dos organismos na zona entre marés (BENEDETTI-CECCHI et al., 1997). Dentre tantas, uma das características físicas mais importantes é a complexidade topográfica de um substrato, particularmente na região entre marés onde a comunidade de macroalgas está sob a pressão mecânica das ondas e sujeita à exposição ao ar (JACOBI et al., 1996). A heterogeneidade do substrato pode modificar o padrão hidrodinâmico específico durante a maré alta influenciando no sombreamento, e na intensidade

1. Departamento de Oceanografia, Universidade Federal de Pernambuco, Av. Arquitetura, s/n, Cidade Universitária, 50.670-901 Recife, PE, Brasil.

2. Instituto de Botânica, Núcleo de Pesquisa em Ficologia, Av, Miguel Estéfano, 3687, 04301-902 São Paulo, SP, Brasil. 
VASCONCELOS. Edson R. T. P. P. de et.al. Padrão espacial da comunidade de macroalgas de mesolitoral em ambiente recifal do nordeste brasileiro.

de vento durante a maré baixa (GUICHARD, et al., 2001). Mesmo assim, é difícil generalizar o papel de cada um dos descritores, pelo grande número de fatores envolvidos e principalmente por suas interações (LITTLE; KITCHING, 1996).

Padrões de zonação em comunidade bentônica já foram bem estudados (STEPHENSON; STEPHENSON 1949; LEWIS, 1964) e o assunto já foi largamente discutido por vários autores (UNDERWOOD, 1981, COUTINHO, 1995, 2002, BOAVENTURA, 2002, GOOD, 2004, MASI; ZALMON, 2008, MASI et al., 2009). No entanto, a maioria dos trabalhos citados se referem ao ambiente de costão rochoso e os trabalhos que se referem a ambientes recifais são escassos (PICHON; MORRISSEY, 1981, PITOMBO et al., 1988).

Os recifes de arenito ou beachrocks são corpos rochosos cimentados por carbonato de cálcio, restritos às regiões tropicais e subtropicais, entre as latitudes $35^{\circ} \mathrm{N}$ e $35^{\circ} \mathrm{S}$ (SWIFT, 1974). No Brasil, os estudos nesses ambientes concentram-se especialmente no litoral nordeste, mas é conhecida a ocorrência destes substratos desde o estado do Ceará até o Rio Grande do Sul. A grande relevância desse tipo de substrato está relacionada ao fato de estar geralmente disposto como cordões ao longo da praia, funcionando como um sistema natural de proteção direta da linha de costa quando não ocorrem reentrâncias, além de servirem como substratos para o desenvolvimento de algas e corais, e também, serem considerados indicadores geológicos da variação do nível do mar (GUERRA e MANSO, 2004). Na costa de Pernambuco e boa parte do litoral nordestino brasileiro, os recifes de franja são considerados como o principal substrato consolidado para a fixação das algas e são constituídos por embasamento arenítico e arenítico-ferruginoso, estando associados frequentemente às algas calcárias e corais, podendo alcançar vários quilômetros de extensão (KEMPF; MORAIS, 1967-69, PEREIRA et al., 2002, 2006).

Gladfelter e Gladfelter (2004) classificaram artificialmente o recife de arenito em três zonas, de acordo com os organismos que o habitam: zona das algas (algal zone), zona dos ouriços (urchin zone) e zona dos corais (coral zone). Cocentino et al., (2004), estudando o macrofitobentos do estado de Pernambuco, destacaram cinco ambientes na estrutura recifal: as cristas que estão mais expostas ao sol e aos ventos; as poças que poderiam ser equivalentes ao ambiente de infralitoral, mas que apresentam características bem marcantes em relação à salinidade e temperatura da água; o platô recifal, local mais plano dos recifes e que, geralmente, ficam expostos ou com apenas uma fina lâmina de água durante as marés baixas; as fendas nas rochas, que abrigam espécies que não suportam luz intensa; e as bordas recifais, onde é encontrada uma maior diversidade de espécies, principalmente rodofíceas e clorofíceas.

O presente estudo tem o objetivo de avaliar a distribuição espacial das macroalgas marinhas e identificar padrões de zonação em um trecho recifal intermareal da praia de Enseada dos Corais, litoral sul de Pernambuco, testando as hipóteses de que a distribuição e a freqüência das macroalgas bentônicas variam de respondem a um gradiente ambiental e se essa distribuição se mantém durante um ciclo anual seco/chuvoso.

\section{MATERIAL E MÉTODOS}

O estudo foi realizado na praia de Enseada dos Corais ( $8^{\circ} 19^{\prime} 08,6^{\prime \prime} \mathrm{S}$ e $34^{\circ} 56^{\prime} 53,1^{\prime \prime} \mathrm{W}$ ), que apresenta cerca de $3,0 \mathrm{~km}$ de extensão, inserida no município do Cabo de Santo Agostinho, litoral sul de Pernambuco. A praia está localizada na região metropolitana do Recife e recebe muitos visitantes durante os fins de semana, principalmente no verão (dezembro a fevereiro) (MARCELINO et al. 2007). O clima é tropical quente e úmido, do tipo As', segundo a classificação de Koppen (ANDRADE; LINS, 1971), ocorrendo dois períodos anuais marcantes: chuvoso(de março a agosto), com precipitação mensal em torno de $100 \mathrm{~mm}$ e o período seco (de setembro a fevereiro). A temperatura média anual do ar fica em torno de $28^{\circ} \mathrm{C}$ e a salinidade da água do mar, em torno de 36 (MACEDO et al., 2004).

A coleta de dados foi feita durante o período seco e chuvoso, em três campanhas amostrais em cada período, nos anos de 2010 e 2011. A amostragem seguiu o método de Line Point Transect descrito por Murray et al., (2001). Os transectos foram montados nas estruturas recifais partindo do andar inferior da região mesolitorânea (após a crista recifal) ao andar 
VASCONCELOS. Edson R. T. P. P. de et.al. Padrão espacial da comunidade de macroalgas de mesolitoral em ambiente recifal do nordeste brasileiro.

médio-superior. Foram traçados 14 transectos de $10 \mathrm{~m}$ de comprimento, distando três metros entre eles, perpendiculares à linha da costa. Foram utilizados 100 pontos regularmente distantes em cada transecto. Os dados foram testados em três grupos de distâncias diferentes: dez grupos de um metro, dois grupos de cinco metros e quatro grupos de 2,5 metros. A melhor compreensão se deu utilizando os grupos de 2,5 metros, sendo assim, os dados foram demonstrados em série de quatro intervalos: grupo I $(0,0$ a 2,5 m); grupo II ( 2,6 a $5 \mathrm{~m})$; grupo III $(5,1$ a $7,5 \mathrm{~m})$ e grupo IV $(7,6$ a $10 \mathrm{~m})$. Em cada um dos pontos, exceto os pontos que ficavam dentro de poças de maré, foi registrada a presença ou ausência das macroalgas, que foram identificadas em campo ao menor nível taxonômico possível. A classificação das espécies seguiu a revisão nomenclatural proposta por Wynne (2011). A frequência relativa das macroalgas foi calculada através do número total de vezes que a espécie aparece na amostra (transecto) dividida pelo total de pontos totais da amostra. A porcentagem média da frequência de relativa foi calculada entre as classes de distância e entre as estações do ano. Foram calculadas também a riqueza de espécie (S) e a diversidade de Shannon $\left(H^{\prime}\right)$ com base logarítmica $e$.

Métodos de estatística multivariada foram usados para as análises quantitativas dos dados de distribuição e abundância das espécies do ambiente estudado. O cálculo de abundância foi feito através do número de vezes em que cada espécie apareceu na amostra (transecto). Foi utilizada uma matriz de similaridade de amostra versus amostra, e criadas três classes de fatores: período do ano, campanha amostral e classe de distância. Para saber se há um padrão de agrupamento das espécies por meio de dados de presença e ausência, foi adotado o índice de Sorensen para formulação da matriz de similaridade. Foi adotado o coeficiente de distância de Bray-Curtis, com o intuito de evidenciar o agrupamento dos organismos em função de sua abundância relativa, pois o mesmo não é afetado por dupla ausência e dar valor significativo a espécies de menor frequência (CLARKE; WARWICK, 2001). Os dados foram transformados pelo $\log (X+1)$, uma vez que no estudo as similaridades podem ser indevidamente dominadas pela soma das poucas espécies de maior frequência, mascarando a importância das espécies menos frequentes na formação dos grupos. As espécies raras (frequência relativa menor que $3 \%$ ) foram retiradas da análise devido ao pouco retorno em termos de poder de detecção dos padrões nas analises multivariadas (Marchant, 2002).

As representações gráficas comparativas dos táxons e sua distribuição nas classes de distância foram feitas através de um Cluster-SIMPROF e um escalonamento multidimensional (MDS), onde os valores de stress (STandardized REsidual Sum of Squares) maiores que 0,2 não são confiáveis (CLARKE, 1993). O teste ANOSIM Two way (análise de similaridade) foi utilizado para refletir a diferença na classificação média das similaridades entre e dentre os grupos definidos a priori. Três hipóteses nulas foram testadas: (i) as espécies de macroalgas podem ser encontradas em qualquer lugar do transeceto de $10 \mathrm{~m}$; (ii) as espécies não apresentam agrupamento em relação a frequência de ocorrência relativa; (iii) não há diferença entre as estações seca/chuvosa do ciclo estudado. A análise de porcentagem de similaridade (SIMPER) definiu o percentual de contribuição dos táxons entre e dentro dos grupos de distâncias analisados (CLARKE; WARWICK, 2001). Foi utilizado um critério de corte de $70 \%$. Os dados foram analisados no software PRIMER-E v6 (CLARKE; GORLEY, 2006).

\section{RESULTADOS E DISCUSSÃO}

\section{Distribuição das espécies dominantes}

Foi identificado um total de 36 táxons de macroalgas, embora apenas quatro tenham apresentado freqüência média total maior que 5\%. Palisada perforata (Bory) K.W. Nam (47,34\% período seco e 58,44 período chuvoso), Ulva Linnaeus, $(11,72 \%$ período seco e $3,83 \%$ período chuvoso), Gelidiella acerosa (Forssk.) Feldmann; Hamel (9,28\% período seco e 10,82\% no período chuvoso), Centroceras Kütz. (8,96\% período seco e 10,76\% período chuvoso). Os táxons Padina Adanson ( $4,15 \%$ período seco e 1,24\% período chuvoso), Sphacelariaceae J.Decaisne (3,11\% período seco e $1,70 \%$ período chuvoso) e Sargassum C. Agardh (2,86\% no período seco e $2,49 \%$ no período chuvoso), mostraram um distinto grau de adaptação ao gradiente de distância analisado. 
VASCONCELOS. Edson R. T. P. P. de et.al. Padrão espacial da comunidade de macroalgas de mesolitoral em ambiente recifal do nordeste brasileiro.

Palisada perforata e Gelidiella acerosa apresentaram distribuição constante, obtendo altos valores médios para as quatro faixas analisadas. Laurencia dendriodea J. Agardh, Padina e Sargassum ficaram restritas às regiões mais próximas ao andar inferior enquanto que Ulva, Sphacelariaceae e Centroceras foram mais freqüentes no andar superior do mesolitoral (Tab. 1).

Tabela 1 Frequência relativa média das macroalgas marinhas bentônicas ao longo de uma faixa $10 \mathrm{~m}$ de comprimento em um recife de arenito intermareal, na praia de Enseada dos Corais Pernambuco.

\begin{tabular}{|c|c|c|c|c|c|c|c|c|}
\hline \multirow[b]{2}{*}{ Estação } & \multicolumn{2}{|c|}{ I (0,0 - 2,5 m) } & \multicolumn{2}{|c|}{ II (2,6 - 5,0 m) } & \multicolumn{2}{|c|}{ III $(5,1$ - 7,5 m) } & \multicolumn{2}{|c|}{ IV $(7,6$ - 10,0 m) } \\
\hline & $\begin{array}{c}\text { Seco } \\
\%\end{array}$ & $\begin{array}{c}\text { Chuvoso } \\
\%\end{array}$ & $\begin{array}{c}\text { Seco } \\
\%\end{array}$ & $\begin{array}{c}\text { Chuvoso } \\
\%\end{array}$ & $\begin{array}{l}\mathrm{Sec} \\
\%\end{array}$ & $\begin{array}{c}\text { Chuvoso } \\
\%\end{array}$ & $\begin{array}{c}\text { Seco } \\
\%\end{array}$ & $\begin{array}{c}\text { Chuvoso } \\
\%\end{array}$ \\
\hline Palisada perforata & 39,71 & 44,32 & 52,84 & 62,53 & 52,94 & 68,38 & 43,86 & 58,52 \\
\hline Ulva sp. & 6,01 & 1,19 & 7,94 & 3,1 & 14,57 & 2,51 & 18,34 & 8,52 \\
\hline Gelidiella acerosa & 11,79 & 11,94 & 11,25 & 11,91 & 4,98 & 7,08 & 9,1 & 12,34 \\
\hline Centroceras sp. & 3,97 & 6,45 & 5,69 & 12.03 & 12,65 & 12,21 & 13,51 & 12,34 \\
\hline Padina sp. & 9,86 & 2,5 & 4,9 & 1,86 & 1,15 & 0,58 & 0,68 & 0 \\
\hline Hypnea sp. & 6,97 & 8,36 & 1,98 & 1,98 & 1,02 & 0,71 & 3,03 & 0,12 \\
\hline Sphacelariaceae & 0,36 & 0 & 2,11 & 0,24 & 4,98 & 3,11 & 5,24 & 3,43 \\
\hline Sargassum sp. & 8,54 & 8,72 & 2,78 & 0,74 & 0,12 & 0,47 & 0 & 0 \\
\hline Acanthophora spicifera & 1,32 & 0,11 & 0,52 & 0,49 & 2,55 & 0,71 & 1,93 & 0,76 \\
\hline Ectocarpus sp. & 0,84 & 1,31 & 2,25 & 0,49 & 0,89 & 0,83 & 0,82 & 0,12 \\
\hline Laurencia dendroidea & 3,00 & 2,38 & 1,32 & 1,11 & 0 & 0 & 0 & 0 \\
\hline
\end{tabular}

Estudos realizados por Ribeiro et al., (2008) em um platô recifal da praia de Boa Viagem mostraram que Palisada perforata (como Chondrophycus papillosus) e Gelidiella acerosa possuem grandes densidades durante $o$ ano, sendo dominantes em ambientes de mesolitoral. Já as algas de distribuição mais restrita são menos tolerantes à exposição ao ar durante a maré vazante, como é o caso de Sargassum sp., Padina sp. e Laurencia dendroidea, que só foram observadas no andar inferior do mesolitoral, ou seja, em um ambiente de menor tempo de emersão. Barradas et al. (2010), estudando a distribuição espacial do macrobentos na praia de Porto de Galinhas, observaram alta cobertura média do recife, em torno de $53 \%$ do total do topo recifal submerso e que os gêneros mais abundantes foram Sargassum, Padina, Caulerpa J.V.Lamouroux e Dictyota J.V.Lamouroux, mostrando, assim, uma forte relação desses gêneros com o ambiente de infralitoral. A figura 1a mostra a distribuição das macroalgas mais frequentes no período seco e a figura 1b, no chuvoso, notando a dominância de Palissada perforata durante todo o ano e ainda uma diminuição da frequência relativa de algumas algas no período chuvoso. 
VASCONCELOS. Edson R. T. P. P. de et.al. Padrão espacial da comunidade de macroalgas de mesolitoral em ambiente recifal do nordeste brasileiro.

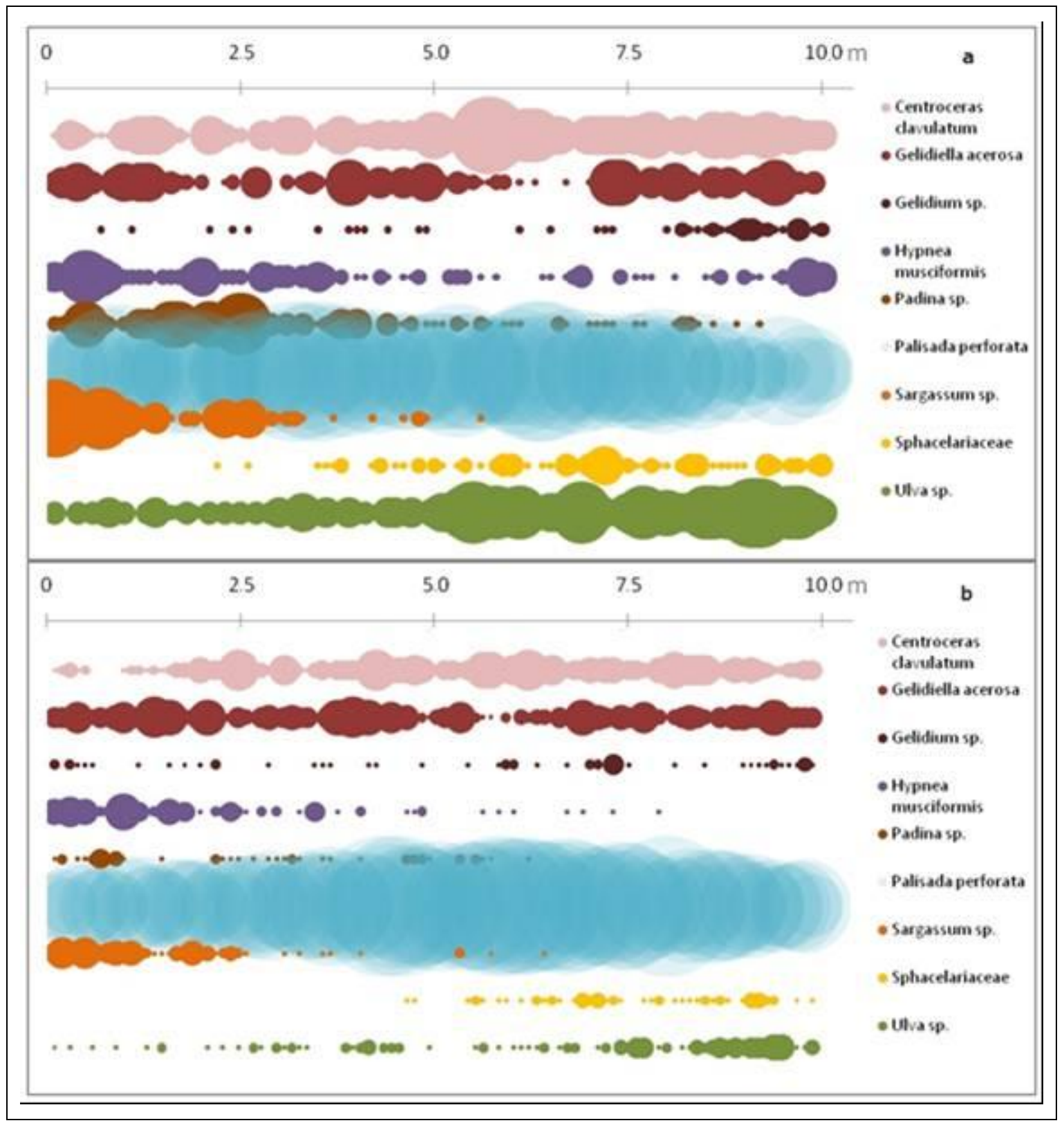

Figura 1 - Esquematização da distribuição das macroalgas ao longo de 14 transectos de 10 metros, no período seco (a) e chuvoso (b), na praia de Enseada dos Corais - Pernambuco, onde o ponto 0 corresponde a área próxima a borda recifal. A largura das bolhas é proporcional à frequência de ocorrência nos pontos indicados.

\section{Padrão de zonação}

As classes de distâncias criadas obtiveram similaridade média acima de $80 \%$ entre si, sendo as espécies Palisada perforata, Centroceras sp., Gelidiella acerosa e Ulva sp. as que mais contribuíram para a similaridade dos grupos (Tab. 2.a). Em relação à diferença entre os grupos, a maior diferença média ocorreu entre o grupo I e IV como esperado, uma vez que esses grupos encontram-se nos extremos opostos do transecto, com dissimilaridade média de 35,2\%. Devido aos grandes valores de frequência de Palisada perforata e Gelidiella acerosa em todas as classes de distâncias, foi preciso utilizar muitas espécies de baixa frequência para explicar a variação entre os grupos. As algas que mais contribuíram para esse resultado foram Sargassum sp., Hypnea sp., Padina sp., Sphacelariaceae e Chaetomorpha sp. em ordem decrescente, totalizando $54,97 \%$ da diferença entre os grupos (Tab. 2.b). Tais algas apresentaram distribuição restrita a uma das duas regiões. A análise SIMPER validou os grupos criados a priori 
VASCONCELOS. Edson R. T. P. P. de et.al. Padrão espacial da comunidade de macroalgas de mesolitoral em ambiente recifal do nordeste brasileiro.

sendo que os mais coesos e diferentes entre si mostraram ainda a existência de um gradiente, uma vez que os grupos vizinhos possuem maior similaridade.

Tabela 1 - Análise SIMPER mostrando a contribuição de cada espécie para a similaridade intra (2.a) e entre os grupos (2.b).

\begin{tabular}{|c|c|c|c|c|c|}
\hline $2 . a$ & Similaridade intra-grupos (\%) & $\bar{I}$ & II & III & IV \\
\hline & Centroceras sp. & 8,68 & 12,19 & 15,02 & 16,41 \\
\hline & Gelidiella acerosa & 11,59 & 13,09 & 10,36 & 14,36 \\
\hline & Hypnea sp. & 6,99 & & & \\
\hline & Laurencia dendroidea & 11,88 & 13,29 & 11,47 & 11,56 \\
\hline & Padina sp. & 8,18 & 8,1 & & \\
\hline & Palisada perforata & 16,66 & 20,4 & 22,29 & 22,94 \\
\hline & Sargassum sp. & 9,16 & & & \\
\hline & Sphacelariaceae & & 8,49 & & \\
\hline & Ulva sp. & & 8,44 & 10,7 & 13,83 \\
\hline & Total & 73,14 & 75,51 & 78,33 & 79,1 \\
\hline & Similaridade média & 85,15 & 80,89 & 81,94 & 81,9 \\
\hline
\end{tabular}

\begin{tabular}{|c|c|c|c|c|c|c|c|}
\hline 2.b & $\begin{array}{l}\text { Dissimilaridade entre os grupos } \\
(\%)\end{array}$ & $\begin{array}{l}\text { I vs } \\
\text { II }\end{array}$ & $\begin{array}{l}\text { I vs } \\
\text { III }\end{array}$ & $\begin{array}{l}\text { I vs } \\
\text { IV }\end{array}$ & $\begin{array}{l}\text { II vs } \\
\text { III }\end{array}$ & $\begin{array}{l}\text { II vs } \\
\text { IV }\end{array}$ & $\begin{array}{l}\text { III vS } \\
\text { IV }\end{array}$ \\
\hline & Acanthophora sp. & 5,14 & & & 7,86 & 5,89 & \\
\hline & Caulerpa sp. & & & & & 10 & \\
\hline & Chaetomorpha sp. & 11,48 & 8,86 & 7,74 & & & \\
\hline & Dictyosphaeria sp. & & & & 6,39 & & \\
\hline & Dictyota sp. & 6,06 & & 4,79 & 5,26 & & \\
\hline & Ectocarpus sp. & 7,03 & 4,64 & & 6,12 & 5,84 & 9,16 \\
\hline & Gelidiella acerosa & & & 5,88 & & 7,63 & \\
\hline & Gelidium sp. & & & & 6,56 & 7,64 & \\
\hline & Gracilaria sp. & 4,85 & 5,7 & & & & \\
\hline & Hypnea sp. & 11,78 & 11,01 & 11,62 & 8,19 & 9,74 & 11,41 \\
\hline & Laurencia dendroidea & 7,21 & 6,23 & 6,12 & & & \\
\hline & Padina sp. & 5,48 & 8,79 & 10,78 & 9,48 & 11,96 & 9,36 \\
\hline & Sargassum sp. & 13,2 & 13,62 & 14,85 & 9,79 & 9,58 & \\
\hline & Sphacelariaceae & 6,31 & 10,93 & 9,98 & 9,15 & 8,39 & 7,2 \\
\hline & Ulva sp. & & & 5,63 & 5,67 & 6,48 & 8,55 \\
\hline & Total & 71,33 & 70,76 & 71,62 & 73,52 & 70,83 & 70,95 \\
\hline & Dissimilaridade média & 21,96 & 31,34 & 35,2 & 22,77 & 27,11 & 17,97 \\
\hline
\end{tabular}

A análise de similaridade two-way (ANOSIM) com 49.999 permutações, revelou diferença entre as estações do ano e ainda entre as classes de distâncias, principalmente entre as de maiores distâncias (Tab. 3). Os testes estatísticos revelaram que a frequência relativa e a composição das espécies de macroalgas variam nas duas estações do ano ( $R$ global de 0,944 
VASCONCELOS. Edson R. T. P. P. de et.al. Padrão espacial da comunidade de macroalgas de mesolitoral em ambiente recifal do nordeste brasileiro.

$p=0,01)$. Os grupos que foram comparados e que atingiram a estatística $R=1$ mostraram que suas amostras são completamente diferentes, ou seja o grupo I é diferente dos grupos III e IV. Os grupos III e IV não apresentaram diferenças significativas entre suas amostras. No MDS (escalonamento multi-dimensional) ficou mais evidente a proximidade entre os grupos de classes vizinhas (Figura 2), e mostram uma diferenciação na composição das amostras do período seco com dois grupos distintos. No período seco verificou-se uma maior frequência das algas em geral, devido ao maior grau de insolação e menor índice pluviométrico médio (CAVALCANTI; KEMPF, 1967-69).

Tabela 2 - ANOSIM, diferença entre os grupos e nível de significância ( $R$ global de 0,707 $\mathrm{p}=0,002)$. Grupo I $(0,0$ a 2,5 m); grupo II $(2,6$ a $5 \mathrm{~m})$; grupo III $(5,1$ a $7,5 \mathrm{~m})$ e grupo IV (7,6 a $10 \mathrm{~m})$.

\begin{tabular}{ccc}
\hline Grupos & R Estatístico & Nível de significância \% \\
I x II & 0,5 & $4^{*}$ \\
\hline I x III & 1 & $1^{*}$ \\
\hline I x IV & 1 & $1^{*}$ \\
\hline II x III & 0,667 & $1^{*}$ \\
\hline II x IV & 0,833 & $1^{*}$ \\
\hline III x IV & $-0,093$ & $66 \mathrm{~ns}$ \\
\hline \multicolumn{2}{c}{${ }^{*} \mathrm{p}<5 \%,(\mathrm{~ns})$ não significativo }
\end{tabular}

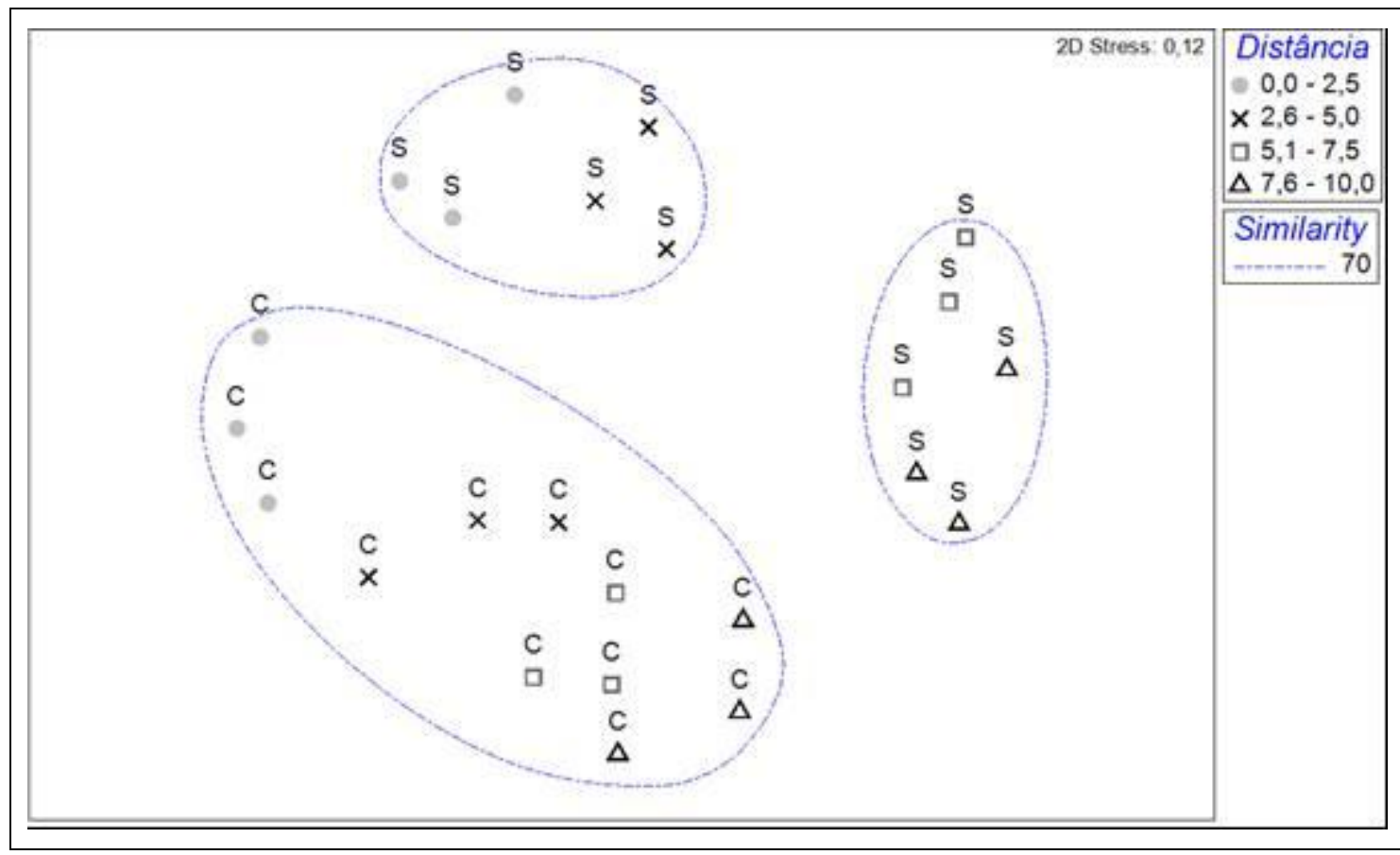

Figura 2 - Escalonamento multidimensional (MDS) baseado no coeficiente de Bray-Curtis, mostrando os grupos de intervalos de distância (I 0-2.5; II 2.6 - 5.0; III 5.1 - 7.5 e IV 7.6 - 10) período do ano: seco (S) e chuvoso (C), com corte em similaridade de $70 \%$.

As macroalgas, em sua maioria, parecem ocorrer em todas as classes de distâncias estabelecidas quando comparadas através do índice de Sorensen de dados de presença e ausência (Figura 3). 
VASCONCELOS. Edson R. T. P. P. de et.al. Padrão espacial da comunidade de macroalgas de mesolitoral em ambiente recifal do nordeste brasileiro.

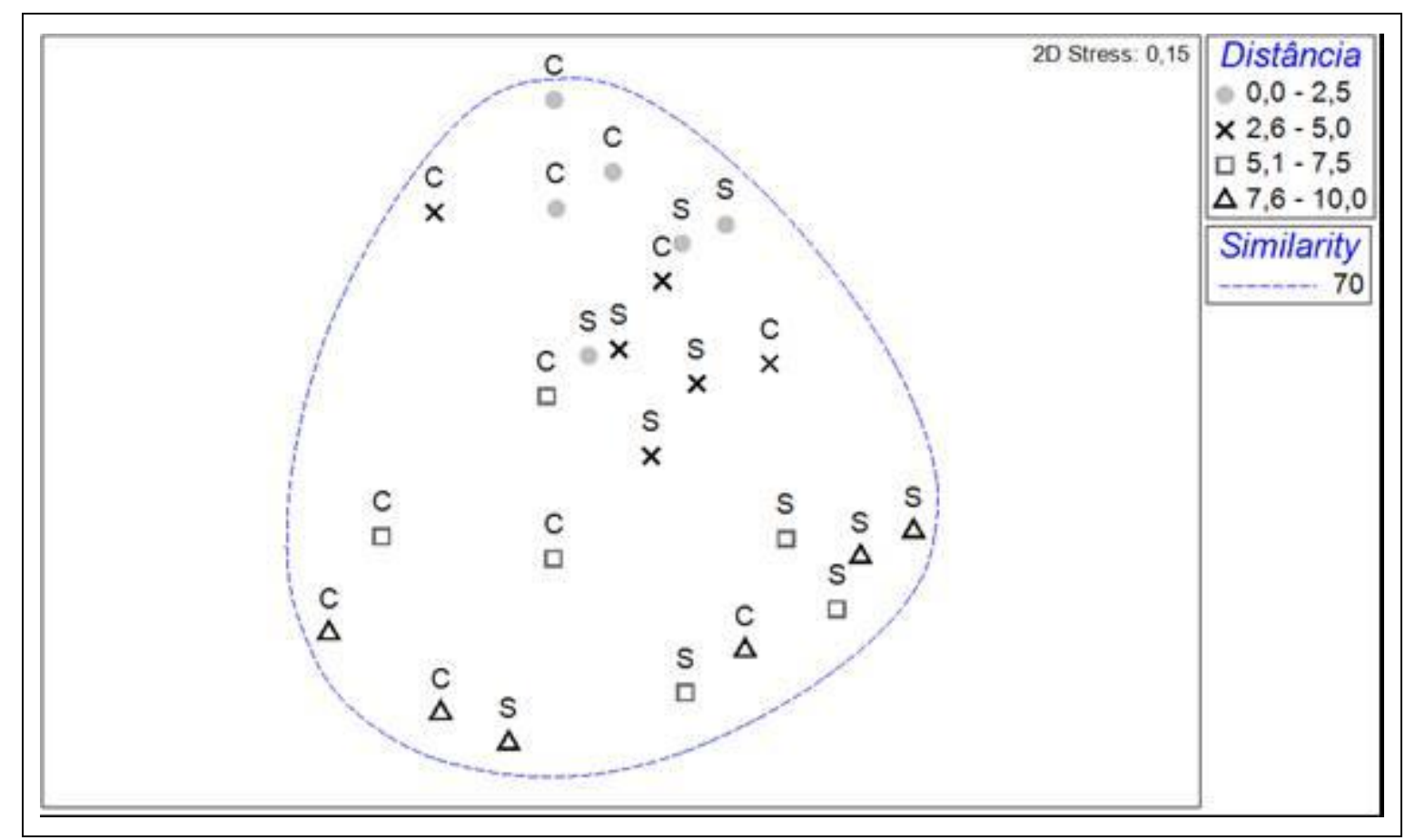

Figura 3 - Escalonamento multidimensional (MDS) baseado no índice de Sorensen, mostrando os grupos de intervalos de distância (I 0-2.5; II 2.6 - 5.0; III 5.1 - 7.5 e IV 7.6 10) período do ano: seco (S) e chuvoso (C), com corte em similaridade de $70 \%$.

Quanto à diversidade e riqueza de espécies, a estação seca apresentou maiores índices em todas as classes de distância em relação ao período chuvoso. Os índices de diversidade foram maiores nas classes de intervalos mais baixas (tabela 4). No desenho amostral proposto, o gradiente de exposição ao ar é maior à medida que aumenta a distancia da borda recifal, tornando assim as classes maiores (de 5,1 a 7,5 e de 7,6 a 10 metros) menos diversas, pois um menor numero de espécies suporta um maior tempo de exposição ao ar. Este resultado está de acordo com as teorias clássicas que explicam a manutenção da diversidade nas quais distúrbios periódicos ajudam a manter a diversidade alta e que a diversidade tende a diminuir em ambientes com alto estresse ambiental (PAINE, 1966, CONNEL, 1970, MENGE, 1976).

Tabela 3. Diversidade média $\left(H^{\prime}\right)$ e riqueza média $(S)$ de espécies. Grupo I $(0,0$ a $2,5 \mathrm{~m})$; grupo II (2,6 a $5 \mathrm{~m})$; grupo III $(5,1$ a $7,5 \mathrm{~m})$ e grupo IV $(7,6$ a $10 \mathrm{~m})$.

\begin{tabular}{ccccc}
\hline Grupos & \multicolumn{2}{c}{ Seco } & \multicolumn{2}{c}{ Chuvoso } \\
& $\mathbf{S}$ & $\mathbf{H}^{`}$ (loge) & $\mathbf{S}$ & $\mathbf{H}^{`}$ (loge) \\
\hline I & 14,3 & 1,90 & 13,3 & 1,7 \\
\hline II & 14,3 & 1,70 & 12,3 & 1,43 \\
\hline III & 11,7 & 1,49 & 11,3 & 1,34 \\
\hline IV & 9,7 & 1,58 & 9 & 1,46 \\
\hline
\end{tabular}

Na praia de Enseada dos Corais, as espécies menos abundantes tais como Sargassum sp., Padina sp., Chaetomorpha sp., Laurencia dendoidea, Hypnea sp. e Ulva sp. foram as que mais participaram na diferenciação dos grupos, embora os estudos clássicos sobre zonação e assembléia de organismos tenham enfocado as espécies mais dominantes (DAVIDSON et al., 2004). 
VASCONCELOS. Edson R. T. P. P. de et.al. Padrão espacial da comunidade de macroalgas de mesolitoral em ambiente recifal do nordeste brasileiro.

A distribuição quali-quantitativa da comunidade de macroalgas nos recifes de Enseada dos Corais evidencia o padrão de duas zonas. A diferença entre tais zonas é mais acentuada no período seco do ano, havendo uma perda desse padrão de distribuição no período chuvoso. Qualitativamente as macroalgas não diferenciam tais zonas, no entanto quantitativamente há uma forte preferencia de determinadas algas habitarem apenas uma delas e até determinada distância, gerando um gradiente de distribuição.

\section{AGRADECIMENTOS}

Ao laboratório de Bentos-Macroalgas da UFPE e toda sua equipe. Aos oceanógrafos Manuele Ataíde e Diego Xavier pelo auxilio nos trabalhos de campo, a CAPES projeto PNADB N.17/2009 pela bolsa e financiamento.

\section{REFERÊNCIAS}

ANDRADE, G. O.; LINS, R. C. Os climas do nordeste. In: VASCONCELOS SOBRINHO J. (Ed.). As Regiões naturais do Nordeste, o meio e a civilização. Recife: CONDEPE, p. 95-138. 1971.

BARRADAS, J.I.; AMARAL, F. D.; ISABEL, M.; HERNANDES, M.; FLORES-MONTES, M. J.; STEINER, A. Q. Spatial distribution of benthic macroorganisms on reef flats at Porto de Galinhas Beach (northeastern Brazil), with special focus on corals and calcified hydroids. Biotemas, Florianopolis v. 23, n. 2, p. 61-67, 2010.

BENEDETTI-CECCHI, L.; CINELLI, F. Spatial distribution of algae and invertebrates in the rocky intertidal zone of the Strait of Magellan: are patterns general? Polar Biology, Kiel, v. 18 n.5, p. 337-343, 1997.

BOAVENTURA, D.; RÉ P., FONSECA, L. C.; HAWKINS, S. J. Intertidal rocky shore communities of the continental portuguese coast: analysis of distribution patterns. Marine Ecology, v. $23 \mathrm{n}$. 1, p. 69-90, 2002.

CAVALCANTI, L.B.; KEMPF, M. Estudo da plataforma continental na área do Recife (Brasil). II. Meteorologia e Hidrologia. Trabalhos Oceanográficos da Universidade Federal de Pernambuco, v9, 10, 11, p.149-158. 1967/69.

CLARKE, K. R., Non-parametric multivariate analyses in community structure. Australian Journal of Ecology. v. 18, p. 117-143. 1993.

CLARKE, K.R.; GORLEY R.N. PRIMER v. 6: User manual/tutorial, PRIMER-E, Plymouth UK, 2006

CLARKE, K.R.; WARWICK, R.M. Change in marine communities: an approach to statistical analysis and interpretation. Plymouth, UK, v. 2, p. 172. 2001.

COCENTINO, A. DE L. M.; MAGALHÃES, K. M., \& PEREIRA, S. M. B. Estrutura do macrofitobentos marinho. In Oceanografia um cenário tropical eds.E. Eskinazi-Leça, $\mathrm{S}$. Neumann-Leitão, \& M. F. Costa. Bagaço. Recife, p. 391-423. 2004.

CONNEL, J. H. A predator-prey system in the marine intertidal region Balanus glandula and several predatory species of Thais. Ecological Monographs. Winter, v. 40 n. 1 p. 49-78. 1970.

COUTINHO, R. Avaliação crítica das causas da zonação dos organismos bentônicos em costões rochosos. Oecologia brasiliensis., v. 1, p. 259-271. 1995.

COUTINHO, R. Bentos de Costões Rochosos. In- Biologia Marinha, eds R. C. Pereira and A. Soares- Gomes. Interciência Press, Rio de Janeiro. p. 147-157. 2002.

$\mathrm{CPRH}$ - Agencia Estadual de Meio Ambiente . Relatório de Classificação das Praias.

Disponível em http://www.cprh.pe.gov.br/monitoramento/balneabilidade/balneabilidade.asp. Acessado em 20 de nov. de 2011.

DAVIDSON I.C.; CROOK A.C.; BARNES D.K.A. Quantifying spatial patterns of intertidal biodiversity: is movement important? Marine Ecology, v. 25, p. 15-34. 2004. 
VASCONCELOS. Edson R. T. P. P. de et.al. Padrão espacial da comunidade de macroalgas de mesolitoral em ambiente recifal do nordeste brasileiro.

GLADFELTER, W. B.; GLADFELTER, E. H. The Beachrock habitat at Southgate Barrier beach: with a comparison to other beachrock habitats on the East End of St. Croix. Coast \& Harbor SCR Technical Report. Gallows Bay, St. Croix. V. 9, p. 5. 2004.

GOOD, T. P. Distribution and abundance patterns in caribbean rocky intertidal zones. Bull. Mar. Sci., v. 74 n. 2, p.459-468. 2004.

GUERRA, N. C., \& MANSO, V. A. V. Beachrocks (Recifes de Arenito). In Oceanografia um cenário tropical eds.E. Eskinazi-Leça, S. Neumann-Leitão, \& M. F. Costa. Bagaço. Recife. p. 109130. 2004.

GUICHARD, F., BOURGET, E. \& ROBERT, J. Scaling the influence of topographic heterogeneity on intertidal benthic communities: alternate trajectories mediated by hydrodynamics and shading. Marine Ecology Progress Series, v. 217, p. 27-41. 2001.

JACOBI, C.M.; LANGEVIN, R., Habitat geometry of benthic substrata: effects on arrival and settlement of mobile epifauna. Journal of Experimental Marine Biology and Ecology, v. 206, p. 39-54. 1996.

KEMPF, M.; MORAIS, J. O. A plataforma continental de Pernambuco (Brasil): Nota preliminar sobre a natureza do fundo. Trabalhos Oceanográficos da UFPE. Recife, v. 9-11, p. 9-26. $1967 / 69$.

LEWIS, J. R. The Ecology of Rocky Shore. English University Press, London, p. 323. 1964. LITTLE, C.; KICHING, J. A. The Biology of Rocky Shores. Oxford University Press, Oxford, p. 241. 1996.

MACEDO, S. J.; MUNIZ, K.; MONTES, M. J. F. Hidrologia da região costeira e plataforma continental do estado de Pernambuco. In Oceanografia um cenário tropical eds.E. EskinaziLeça, S. Neumann-Leitão, \& M. F. Costa. Bagaço. Recife, p. 255-268. 2004.

MARCELINO, B. F.; RACCAH, L. A.; ANJOS, F. B. R. D.; ARAÚJO, R. L. \& RODRIGUES, K. A. D. S. Mapeamento de algas e ouriços no Recife de Enseada dos Corais - PE, utilizado na gestão do ecoturismo local. Anais do VI Congresso Nacional de Ecoturismo (Vol. Itatiaia). 2007.

MARCHANT, R. Do rare species have any place in multivariate analysis for bioassessment? Journal of the North American Benthological Society, v. 21, p. 311-313. 2002.

MASI, B. P.; MACEDO, I. M.; ZALMON, I. R. Benthic Community Zonation in a Breakwater on the North Coast of the State of Rio de Janeiro, Brazil. Brazilian Archives of Biology and Technology, v. 52 n3, p. 637-646. 2009.

MASI, B. P.; ZALMON, I. R. Zonação de comunidade bentica do entremarés em molhes sob diferente hidrodinamismo na costa norte do estado do Rio de Janeiro, Brasil. Revista Brasileira de Zoologia, v. 25 n. 4, p. 662-673. 2008.

MENGE, B. A. Organization of the New England rocky intertidal community: role of predation, competition, and enviromental heterogeneity. Ecol. Monogr. V.46, p. 355-393. 1976.

MURRAY, S.N.; AMBROSE, R.F.; DETHIER, M.N., Methods for Performing Monitoring,

Impact, and Ecological Studies on Rocky Shores. eds S. N. Murray, R. F. Ambrose, \& M. N. Dethier, Santa Barbara, California Coastal Research Center, Marine Science Institute, University of California. 2001.

PAINE, R. T. Food web complexity and species diversity. Am. Nat. V. 100, p. 67-75. 1966.

PEREIRA, S. M. B. Desenvolvimento e situação atual do conhecimento das macroalgas marinhas das regiões nordeste e norte. In: Biodiversidade, conservação e uso sustentável da flora do Brasil. eds. E. L. Araújo, A. N. Moura, E. V. S. B. Sampaio. Universidade Federal Rural de Pernambuco. Recife, p. 117-121. 2002.

PEREIRA, S. M. B.; OLIVEIRA-CARVALHO, M. D. F.; BURGOS, D. C.; ARAÚJO, E. DE L. Caracterização estrutural das macroalgas de ambiente recifal da praia de Enseada dos Corais - 
VASCONCELOS. Edson R. T. P. P. de et.al. Padrão espacial da comunidade de macroalgas de mesolitoral em ambiente recifal do nordeste brasileiro.

Pernambuco - Brasil. Congresso Brasileiro de Ficologia, 11 Simpósio Latino-Americano sobre algas nocivas. Museu Nacional Rio de Janeiro. Itajaí. P. 231-242. 2006.

PICHON, M.; MORRISSEY, J.. Benthic zonation and community structure of south island reef, lizard island (Grea t barrier reef). Bulletin of Marine Science, v. 31, n. 3, p. 581-593. 1981.

PITOMBO, F. B.; RATTO, C. C.; BELÉM, M. J. C. Species diversity and zonation pattern ofhermatypic coraIs at two ftinging reefs of Abrolhos Archipelago, Brazil. In: Choat, J. H.et ai. eds. Proceedings of the 6th International Coral Reef Symposium, v. 2, p. 817-820. 1988.

RIBEIRO, F. A.; TRAVASSOS JÚNIOR, A.; GESTINARI, L. M.; TORRES, J.; LIMA, K. K. D. A.; SANTOS, M. D. D.; LIRA, G. A. S. T. et al. Análise Quali-Quantitativa Das Populações Algáceas De Um Trecho Recifal Na Praia De Boa Viagem, PE. Oecologia brasiliensis. v. 12, n. 02, p. 222-228. 2008.

SABINO, C. M.; VILLAÇA, R. Estudo comparativo de métodos de amostragem de comunidades de costão. Brazilian Journal of Biology. v. 59, p. 407-419. 1999.

STEPHENSON, T. A.; STEPHENSON, A. The Universal feature of zonation between tide-marks on rocky coasts. J. Ecol., v. 37, p. 289-305. 1949.

SWIFT, D. J. P. Continental shelf sendimatation. In: Geology of Continental Margins. eds. C. A. Burk, C. L. Drake. Spring -Verlag. Berlim. p. 117-136. 1974.

UNDERWOOD, A. J. Structure of a rocky intertidal community in New South Wales: Patterns of vertical distribution and seasonal changes. Journal of Experimental Marine Biology and Ecology, v. 51 n. 7, p. 57-85. 1981.

WYNNE, M. J. A checklist of benthic marine algae of the tropical and subtropical western Atlantic: third revision. Nova Hedwigia, Beiheft v. 129, p. 1-153. 2011. 